\title{
CORRESPONDENCE \\ Kicking it through the uprights: getting it published after presenting at PAS
}

\author{
Pediatric Research (2021) 89:1598-1600; https://doi.org/10.1038/s41390- \\ 020-01182-y
}

\begin{abstract}
INTRODUCTION
The Pediatric Academic Society (PAS) annual meeting provides opportunities for investigators to present research, exchange ideas, and develop collaborations. For pediatric emergency medicine researchers, PAS is the premier scientific meeting in the United States and globally. Submitted abstracts are reviewed by a panel of experts and, if selected for inclusion in the meeting, are assigned to be presented as a poster or as an oral presentation (platform) based on reviewers' scores. A platform or poster at PAS is a major milestone in the life of a research project, but the project is not considered complete until the manuscript is published. Prior studies have examined the rates of manuscript publication after presentations at meetings, but none have focused specifically on pediatric emergency medicine. ${ }^{1}$

When the 2020 PAS annual research meeting was cancelled due to the coronavirus disease 2019 (COVID-19) pandemic, the opportunity to present accepted research was removed, with uncertain impacts on later publication. To judge the impact of this cancellation on publication rates, we wanted to establish a baseline publication rate for accepted abstracts. To this end, we determined the number of pediatric emergency medicine abstracts accepted for meeting presentation that had been published in the peer-reviewed journals within 3 years of the 2017 PAS meeting.
\end{abstract}

\section{METHODS}

Design

We performed a retrospective cohort study using publicly available data. The study protocol was deemed exempt by the Boston Children's Hospital Institutional Review Board.

\section{Selection of abstracts}

We identified accepted abstracts to the 2017 PAS meeting using the online program guide and selected those abstracts with emergency medicine as the primary category. ${ }^{2}$ We then extracted the following from each presentation: author names, number of authors, number of author institutions, abstract title, and presentation format (poster or platform).

\section{Assessment of subsequent publication}

We then determined the publication status for each of the identified pediatric emergency medicine abstracts using the PubMed index. We searched using the last names of the first and last authors and considered a publication a match if both the authors and the topic agreed. For each match, we abstracted the journal name and date of print publication. For journals where publication is less frequent than monthly, we defined the publication date as the first month of the publication interval. We determined the journal impact factor by year using the Web of Science citation index. The most recent impact factor released in 2019 reflects 2018 citations.

\section{Data analysis}

Nominal variables (such as presentation format) were compared using the Chi-square test. Continuous variables (such as number of institutions) were compared using Mann-Whitney test. Time to an event (manuscript publication) was described with a Kaplan-Meier curve and comparison between groups was analyzed by using Log-rank test. Multiple linear regression was used to establish which factors remained independently associated with the outcome when adjusted for the others. We dichotomized the number of author institutions ( $\leq 2$ vs. $\geq 3$ ) to best indicate multi-institutional involvement in the research project.

We used SPSS version 23.0.0 (IBM SPSS Software; Armonk, NY) and JMP Pro 14.0 (SAS Institute Inc., Cary, NC) for all analyses.

\section{RESULTS}

Of the 378 pediatric emergency medicine abstracts submitted to the 2017 PAS meeting, 301 (79.6\%) were accepted for presentation at the meeting. Of these, $32 / 301$ (10.6\% of accepted) were selected for platform and 269/301 (89.4\%) for poster presentations. For accepted abstracts, the median number of authors was 5 [interquartile range (IQR) 4-7 authors) and author institutions was 1 (IQR 1-7 institutions).

Overall, $150 / 301$ (49.8\% of accepted pediatric emergency medicine abstracts) were published within 3 years of the PAS meeting presentation. Platform presentations were more likely to be published than posters (22/32, $68.8 \%$ platforms vs. $128 / 269$, $47.6 \%$ posters, $p<0.001$; Table 1 ).

Of the 52 different journals where these abstracts were published, the eight most frequent were Pediatric Emergency Care $(n=30$ publications), Pediatrics $(n=16)$, Prehospital Emergency Care $(n=8)$, Academic Emergency Medicine $(n=7)$, American Journal of Emergency Medicine $(n=7)$, Annals of Emergency Medicine $(n=7)$, Journal of Pediatrics $(n=7)$, and Academic Pediatrics $(n=6)$. The highest impact journals were New England Journal of Medicine $(n=1)$ and Lancet $(n=1)$.

For published pediatric emergency medicine abstracts, the median time from PAS meeting presentation (May 2017) until manuscript publication was 17 months (IQR 10-26 months). Using Kaplan-Meier survival analysis, platforms had a shorter time to publication than posters $(p=0.003$; Fig. 1$)$. The impact factor of the journals ranged from 0.6 to 70.7 (2.6, IQR 1.2-3.7) and was higher for platform than for poster presentations (median 5.4 IQR $1.4,5.5$ platforms vs. 2.3 IQR 1.2, 3.2 posters; $p=0.006$ ).

Both selection for platform presentation [ $\beta$-coefficient $5.9,95 \%$ confidence interval (Cl) 2.8-9.2] and three or more author

These authors contributed equally: Sophia E. Nigrovic, Henry D. Fine.

Received: 13 May 2020 Revised: 14 August 2020 Accepted: 24 August 2020

Published online: 1 October 2020 
Table 1. Comparison between published and not yet published abstracts accepted to the 2017 PAS meeting $(n=301)$.

$\begin{array}{llll}\text { Published } & \text { Not yet } & \text { Total } & p \text { Value } \\ N=150 & \text { published } & N=301 & \\ & N=151 & & \end{array}$

\begin{tabular}{|c|c|c|c|c|}
\hline \multicolumn{5}{|l|}{ Format } \\
\hline Platform & $22(68.8 \%)$ & $10(31.2 \%)$ & 32 & 0.001 \\
\hline Poster & $128(47.6 \%)$ & $141(52.4 \%)$ & 269 & \\
\hline Number of authors ${ }^{a}$ & $6(4,8)$ & $5(4,7)$ & $5(4,7)$ & 0.002 \\
\hline \multicolumn{5}{|c|}{ Number of institutions } \\
\hline 1 or 2 institutions & $114(46.3 \%)$ & 132 (53.7\%) & 246 & 0.01 \\
\hline$\geq 3$ institutions & $36(65.5 \%)$ & 19 (34.5\%) & 55 & \\
\hline $\begin{array}{l}\text { Number of } \\
\text { institutions }{ }^{a}\end{array}$ & $1(1,2)$ & $1(1,2)$ & & 0.001 \\
\hline
\end{tabular}

${ }^{\text {a }}$ Median (interquartile range).

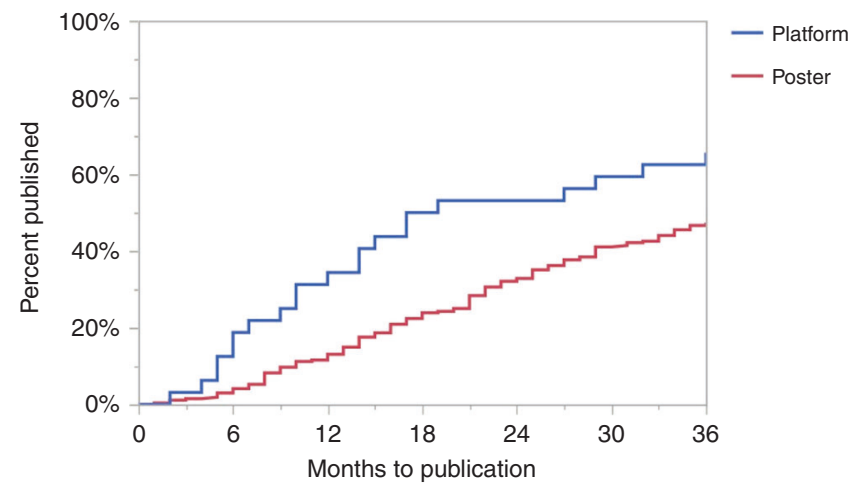

Fig. 1 Time to publication from 2017 PAS meeting for pediatric emergency medicine abstracts. Kaplan-Meier curve demonstrates the percentage of accepted abstracts published by month stratifed by platform (blue line) and poster (red line).

institutions ( $\beta$-coefficient $4.1,95 \% \mathrm{Cl} 1.5-6.8$ ) were independently associated with higher journal impact factor.

\section{DISCUSSION}

Three years after the 2017 PAS meeting, half of the accepted pediatric emergency medicine abstracts have been published in the peer-reviewed literature, with higher rates for platform presentations. Both selection as a platform and involvement of authors from multiple institutions were associated with publication in higher impact journals. Meeting planners select the highest rated abstracts for platform presentations and the work to prepare for presentation may facilitate manuscript preparation. Additionally, sharing ideas, data, and expertise across multiple institutions can improve the ability to address important questions that may not be possible in a single-center study.

Publication rates following presentation at national conferences vary widely from 5 to $90 \%$ based on the type of conference and the duration of follow-up. ${ }^{3-6}$ A 2018 Cochrane review included 425 studies examining abstract publication rates after scientific meetings from a variety of subject areas. ${ }^{1}$ Overall, $47 \%$ of abstracts were published in under 10 years, with $35 \%$ within the first 3 years. In a study of randomly selected PAS general pediatric abstracts from the 1998 and 1999 meetings, 46\% of selected abstracts were published within 5 years. ${ }^{7}$ Our study, the first focused exclusively on pediatric emergency medicine abstracts and the most recent look at the PAS meeting, had a slightly higher publication rate within our 3-year time window.

After nearly 90 years of annual PAS meetings, the cancellation due to COVID-19 is unprecedented. The impact of this cancellation on the pediatric science selected for presentation is yet unclear. On the one hand, the rate of publication from the abstracts selected for the 2020 meeting may decline as authors no longer had a set deadline for completing analyses and for preparing presentations, which often serve as manuscript templates. Additionally, reduced collaboration and feedback as well as idea exchange that occurs in the meeting halls may slow project completion. On the other hand, the rate of publication may increase if authors use the time saved from presentation preparation and meeting attendance to draft their scientific manuscript. On a personal note, the idea for this manuscript was born after this study's first authors had a premature end to their in-person senior year of high school as the COVID-19 pandemic mandated a shift to remote learning. The authors were able to divert some focus from their regular classes to pursue this project.

Our study has several limitations. Since only 3 years have passed since the 2017 PAS meeting, we could not capture all publications including those currently in press (accepted for publication but not yet PubMed indexed). However, the Cochrane review demonstrated that the vast majority of meeting abstracts successfully published reach print journals within 3 years of presentation. In fact, many university promotions committees suggest a faculty member remove unpublished abstracts from a curriculum vitae after three years, highlighting the importance of timely publication. Second, although we used an exhaustive search strategy, we still may have missed some publications with our search strategy because of name changes (first or last authors) or publication in journals not yet indexed by Pubmed. Third, we have overestimated the number of author institutions. In some cases, an author may have moved from one institution to another between data collection and meeting presentation (e.g., a graduating trainee accepting a new position). Fourth, impact factors are updated annually but are not yet available for 2019 and 2020 and may have changed incrementally but unlikely to have substantially impacted this analysis. Last, we only analyzed 1 year of pediatric emergency medicine PAS presentations and publications rates may vary between years.

As many abstracts take years to be published as full manuscripts, scientific meetings provide a valuable forum to share preliminary findings. However, manuscript publication ${ }^{8}$ allows both additional scientific scrutiny in the form of peer review and widespread, long-lasting dissemination of findings. Half of the 2017 accepted pediatric emergency medicine abstracts have not yet been published, reflecting lost opportunities for sustained knowledge sharing. After the cancellation of the 2020 PAS meeting, we will need to closely track the impact of this lost opportunity on subsequent manuscript publication.

\section{ADDITIONAL INFORMATION}

Competing interests: The authors declare no competing interests.

Publisher's note Springer Nature remains neutral with regard to jurisdictional claims in published maps and institutional affiliations.

Sophia E. Nigrovic ${ }^{1}$, Henry D. Fine ${ }^{2}$, Lise E. Nigrovic ${ }^{3}$ and Andrew M. Fine ${ }^{3}$

${ }^{1}$ Brookline High School, Brookline, MA, USA; ${ }^{2}$ Buckingham Browne and Nichols School, Cambridge, MA, USA and ${ }^{3}$ Emergency Medicine, Boston Children's Hospital, Boston, MA, USA These authors contributed equally: Sophia E. Nigrovic, Henry D. Fine. Correspondence: Andrew M. Fine (Andrew.Fine@childrens.harvard. edu) 


\section{REFERENCES}

1. Scherer, R. W. et al. Full publication of results initially presented in abstracts. Cochrane Database Syst. Rev. MR000005 (2018). Cited 5 May 2020.

2. PAS. Pediatric Academic Societies Meeting, May 6-9, 2017, San Francisco, CA. https://www.xcdsystem.com/pas/program/2017/ (2017). Cited 11 May 2020.

3. Hoag, C. C., Elterman, D. S. \& MacNeily, A. E. Abstracts presented the American Urological Association Annual Meeting: determinants of subsequent peer reviewed publication. J. Urol. 176, 2624-2629 (2006).

4. Peng, P. H., Wasserman, J. M. \& Rosenfeld, R. M. Factors influencing publication of abstracts presented at the AAO-HNS Annual Meeting. Otolaryngol. Head Neck Surg. 135, 197-203 (2006).
5. O'Dell, K. M. \& Shah, S. A. Evaluation of pharmacy practice residents' research abstracts and publication rate. J. Am. Pharm. Assoc. 52, 524-527 (2012).

6. Tam, V. C., Tannock, I. F., Massey, C., Rauw, J. \& Krzyzanowska, M. K. Compendium of unpublished phase III trials in oncology: characteristics and impact on clinical practice. J. Clin. Oncol. 29, 3133-3139 (2011).

7. Carroll, A. E., Sox, C. M., Tarini, B. A., Ringold, S. \& Christakis, D. A. Does presentation format at the Pediatric Academic Societies' Annual Meeting predict subsequent publication? Pediatrics 112, 1238 (2003).

8. Gottlieb, M., Davenport, D., Schiebout, J., Treviño, R. R. \& Purim-Shem-Tov, Y. A. Percentage of studies presented at a conference prior to publication in emergency medicine journals. Acad. Emerg. Med. https://doi.org/10.1111/acem.13911 (2019). Cited 22 Jul 2020. 